\title{
Ventricular Repolarization Dispersion During Ischemia Course Measured by Temporal and Spatial Electrocardiographic Parameters
}

\author{
PD Arini ${ }^{1,2}$, FH Baglivo ${ }^{2}$, JP Martínez ${ }^{3,4}$, P Laguna $^{3,4}$ \\ ${ }^{1}$ Instituto Argentino de Matemática, CONICET, Buenos Aires, Argentina \\ ${ }^{2}$ Facultad de Ingeniería, Universidad de Buenos Aires, Buenos Aires, Argentina \\ ${ }^{3}$ Instituto de Investigación en Ingeniería de Aragón, Universidad de Zaragoza, Zaragoza, Spain \\ ${ }^{4}$ CIBER de Bioingeniería, Biomateriales y Nanomedicina (CIBER-BBN), Spain
}

\begin{abstract}
In this work we studied the evolution of increased ventricular repolarization dispersion (VRD) in the ECG during ischemia induced by Percutaneous Transluminal Coronary Angioplasty (PTCA). T-wave duration is measured by an automatic delineator and proposed as a index to quantify increased VRD. Moreover, spatial complexity of repolarization is measured by Singular Value Decomposition (SVD) related indexes. Results showed a statistically significant widening of T-wave peak-to-end during PTCA procedure in RCA and LAD occluded patients. Besides, a statistically significant dynamic change of the dipolar components was observed during PTCA in RCA, LAD and LCX occluded patients. On the other hand, the relative energy of non-dipolar components did not change during the time course of increased VRD evolution.
\end{abstract}

\section{Introduction}

The transmural acute ischemia is accompanied by a succession of electrophysiological changes in the process of cardiac ventricular repolarization. That is a complex process of the balance between conduction slowing on the one hand and action potential duration (APD) shorthening on the other. Therefore acute ischemia is considered a risk factor, because the probability to suffer sudden cardiac death is augmented in conditions of increased VRD. We hypothesized that at early ischemia stages the endocardium APD is slightly reduced or unaltered while the epicardium one is reduced in a larger proportion. This has been previously reported in isolated canine ventricular cardiac tissue studies [1]. These authors suggested that the presence of $I_{t o}$ (transient outward potassium current) in epicardium but not endocardium contributes importantly to the selective electrical depression by induced ischemia.
On the other hand an increased VRD implies a modification of the T-wave morphology evidenced on the surface ECG. Furthermore, new experimental studies have shown that T-wave widening could be a marker of increased VRD [2]. Ballon-inflation PTCA is an advantageous model to provide ischemia in humans due to a total interruption of blood flow through a coronary artery providing a localized ischemic region at the ventricles. Hence the aim of this work is to study the evolution of VRD in the course of transmural ischemia induced by PTCA procedure. For this purpose, we computed ECG indexes to quantify VRD based on both, temporal repolarization dispersion (TRD) and spatial complexity of repolarization (SCR) parameters.

TRD indexes are T-wave peak to end $\left(T_{\mathrm{PE}}\right)$ and T-wave width $\left(T_{\mathrm{W}}\right)$ [2]. SCR indexes are the ratio of the $2^{\text {nd }}$ to the $1^{\text {st }}$ eigenvalues $\left(T_{\mathrm{C}-2 \mathrm{D}}, 2 \mathrm{D}\right.$ T-wave complexity) in the repolarization SVD [3], the ratio of the $2^{\text {nd }}$ eigenvalue to the total energy ( $T_{\mathrm{C}-2 \mathrm{nd}}$, second order T-wave complexity) [3] and the ratio of the energy of non-dipolar components to the total energy ( $T_{\mathrm{wR}}, \mathrm{T}$-wave residuum) [4].

\section{Methods}

\subsection{Data set}

The study group consisted of 93 ECG records from patients at the Charleston Area Medical Center in West Virginia undergoing elective prolonged balloon occlusion during PTCA in one of the major coronary arteries (STAFF-III study) [5]. This group was selected from a total of 108 patients, with the condition that T-wave could be delineated during the complete time course of ischemia. The mean inflation duration was 4' 28 "' with a standard deviation of 74 ". Eight leads $\left(\mathrm{V}_{1}-\mathrm{V}_{6}\right.$, I, II) were recorded using equipment by Siemens-Elena $\mathrm{AB}$ (Solna, Sweden) and digitized at sampling rate of $1000 \mathrm{~Hz}$ and amplitude resolution of $0.6 \mu \mathrm{V}$. Leads III, aVR, aVL and aVF were 


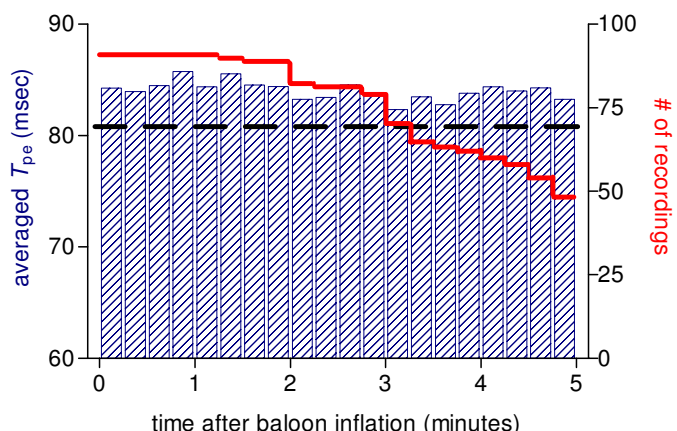

(a)

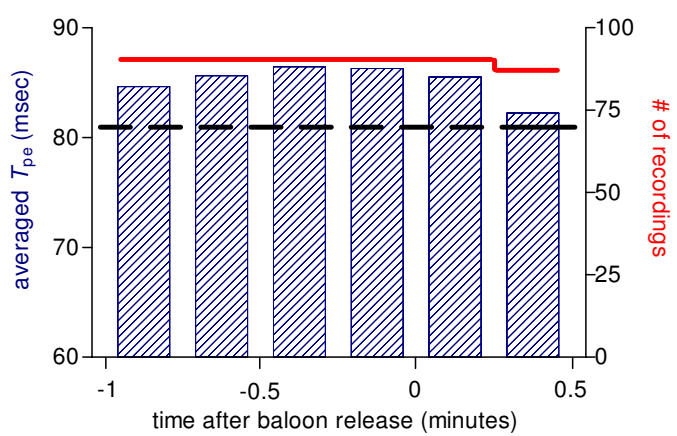

(b)

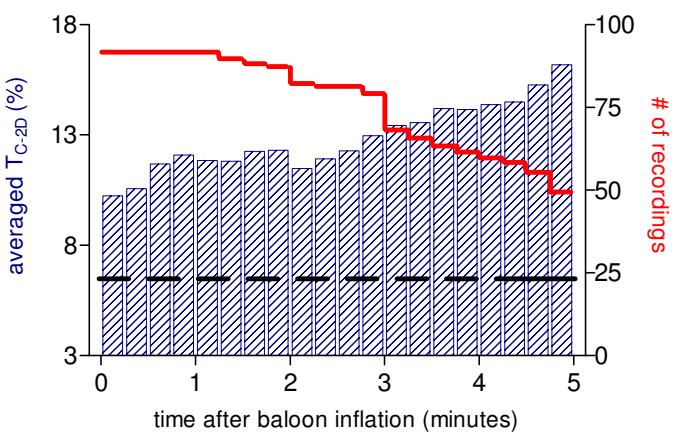

(c)

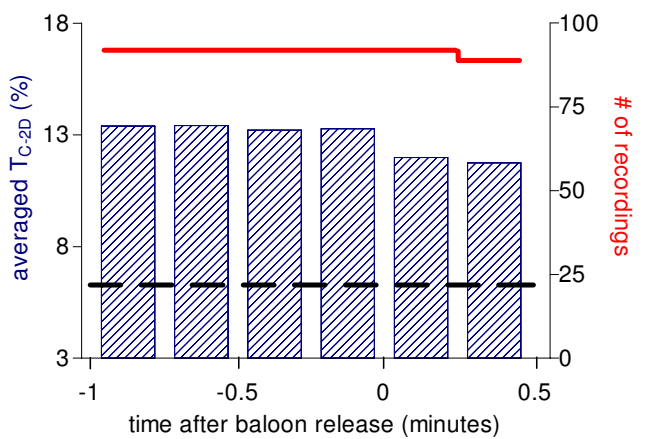

(d)

Figure 1. Mean value (left axis) of $T_{\mathrm{PE}}$ (a) and $T_{\mathrm{C}-2 \mathrm{D}}$ (c) at a given time relative to the balloon inflation associated with the corresponding number of recordings (right axis). Mean value (left axis) of $T_{\mathrm{PE}}(\mathrm{b})$ and $T_{\mathrm{C}-2 \mathrm{D}}(\mathrm{d})$ relative to the balloon release (between one minute before and half a minute after the release) associated with the corresponding number of recordings (right axis). The dotted horizontal line indicates the mean value measured at control situation.

derived from leads I and II. Synthesized orthogonal XYZ leads were also obtained. Two ECG were acquired for each patient in supine position. First, a control 5-minute ECG was recorded some time before the PTCA procedure and the second ECG was recorded during PTCA procedure. The occlusion sites were also considered separately for this study: left anterior descending (LAD) coronary artery in 29 patients, right coronary artery (RCA) in 45 patients and left circumflex (LCx) coronary artery, in 19 patients.

\subsection{ECG processing}

For a total of 93 patients, 15 ECG leads were processed in control situation and PTCA procedure respectively. First, QRS fiducial points were detected on a multilead basis by an automatic QRS detector [6]. Secondly, cubic spline interpolation was used for baseline wander rejection. Finally, ECG delineation system based on the wavelet transform has been used for T-wave location and delineation [7]. Six recordings ( 2 from LAD, 1 from LCX and 3 from RCA group) presented an extremely low SNR in some leads. In those cases we only considered delineation on leads I, II, $\mathrm{V}_{1}, \mathrm{~V}_{2}$ and $\mathrm{V}_{3}$.

\subsection{ECG repolarization parameters}

To quantify and analyze TRD at each $i^{t h}$ beat, the indexes $T_{\mathrm{w}}$ and $T_{\mathrm{PE}}$ were calculated. We applied a multilead algorithm for the final T-wave width as:

$$
T_{\mathrm{w}, \mathrm{i}}=T_{\text {end, } \mathrm{i}}-T_{\text {onset, } \mathrm{i}}
$$

and T-wave peak-to-end duration as:

$$
T_{\mathrm{PE}, \mathrm{i}}=T_{\text {end, } \mathrm{i}}-T_{\text {peak, } \mathrm{i}}
$$

To estimate the onset and end it is selected between the 15 leads one $T_{\text {onset }}$ (the earliest reliable T-wave onset at 
Table 1. Mean values \pm SEM of VRD indexes at different stages of the PTCA procedure. ${ }^{*} \mathrm{p}<0.05,{ }^{* *} \mathrm{p}<0.01,{ }^{\dagger} \mathrm{p}<0.005$, ${ }^{\ddagger} \mathrm{p}<0.001,{ }^{\S} \mathrm{p}<0.0005,{ }^{\#} \mathrm{p}<0.0001$ vs. control situation.

\begin{tabular}{|c|c|c|c|c|c|c|c|}
\hline & Occluded artery & $D_{\text {CONTROL }}$ & $D_{\mathrm{PTCA}}^{\mathrm{OS}}$ & $D_{\mathrm{PTCA}}^{\mathrm{O} 1}$ & $D_{\text {PTCA }}^{\mathrm{O} 2}$ & $D_{\text {PTCA }}^{\mathrm{O} 3}$ & $D_{\text {PTCA }}^{\mathrm{OE}}$ \\
\hline \multirow{3}{*}{$T_{\mathrm{W}}(m s)$} & LAD & $221.0 \pm 8.4$ & $220.9 \pm 8.8$ & $224.3 \pm 8.9$ & $218.3 \pm 9.1$ & $208.1 \pm 9.9$ & $212.8 \pm 8.6$ \\
\hline & RCA & $220.5 \pm 4.0$ & $238.6 \pm 5.1^{\dagger}$ & $234.2 \pm 5.2 *$ & $230.5 \pm 4.7^{*}$ & $226.6 \pm 4.6$ & $225.6 \pm 4.8$ \\
\hline & LCX & $234.2 \pm 6.6$ & $223.1 \pm 6.3$ & $217.2 \pm 7.3$ & $216.9 \pm 7.8$ & $230.8 \pm 9.7$ & $230.4 \pm 8.1$ \\
\hline \multirow{3}{*}{$T_{\mathrm{PE}}(m s)$} & LAD & $80.1 \pm 3.1$ & $84.9 \pm 3.0 *$ & $88.1 \pm 3.6^{*}$ & $89.8 \pm 3.8^{*}$ & $85.9 \pm 2.4 *$ & $89.2 \pm 4.3 *$ \\
\hline & RCA & $79.2 \pm 1.7$ & $92.5 \pm 3.9 \ddagger$ & $88.2 \pm 3.4^{\dagger}$ & $85.7 \pm 2.4^{* *}$ & $89.6 \pm 3.5 *$ & $88.1 \pm 3.2 * *$ \\
\hline & LCX & $85.3 \pm 3.2$ & $81.9 \pm 2.7$ & $85.7 \pm 2.3$ & $86.5 \pm 3.2$ & $92.2 \pm 3.6$ & $91.2 \pm 4.3$ \\
\hline \multirow{3}{*}{$T_{\mathrm{C}-2 \mathrm{D}}(\%)$} & LAD & $6.8 \pm 0.9$ & $11.3 \pm 1.2^{\dagger}$ & $11.9 \pm 1.8^{* *}$ & $10.8 \pm 1.6^{*}$ & $11.3 \pm 1.7^{*}$ & $10.1 \pm 1.6$ \\
\hline & RCA & $5.8 \pm 1.1$ & $9.6 \pm 1.9$ & $10.7 \pm 1.9^{\dagger}$ & $11.5 \pm 1.9 \S$ & $12.8 \pm 1.9 \S$ & $14.4 \pm 2.2 \#$ \\
\hline & LCX & $8.5 \pm 2.9$ & $10.2 \pm 1.8^{*}$ & $13.7 \pm 2.5 * *$ & $12.5 \pm 2.0 *$ & $13.6 \pm 2.4^{* *}$ & $16.3 \pm 3.0^{* *}$ \\
\hline \multirow{3}{*}{$T_{\mathrm{C}-2 \mathrm{nd}}(\%)$} & LAD & $6.2 \pm 0.8$ & $9.8 \pm 1.0^{\dagger}$ & $10.2 \pm 1.4^{*}$ & $10.7 \pm 1.8^{*}$ & $10.0 \pm 1.3^{*}$ & $8.7 \pm 1.3$ \\
\hline & RCA & $4.8 \pm 0.8$ & $7.4 \pm 1.3^{*}$ & $8.3 \pm 1.2^{\dagger}$ & $8.9 \pm 1.1$ \# & $10.0 \pm 1.2 \#$ & $11.0 \pm 1.4 \#$ \\
\hline & LCX & $6.8 \pm 1.9$ & $8.6 \pm 1.4^{*}$ & $11.8 \pm 1.7^{\dagger}$ & $11.0 \pm 1.5^{*}$ & $11.7 \pm 1.7$ * & $12.5 \pm 2.0 * *$ \\
\hline \multirow{3}{*}{$T_{\mathrm{WR}}(\%)$} & LAD & $0.2 \pm 0.1$ & $0.4 \pm 0.1$ & $0.4 \pm 0.1$ & $0.3 \pm 0.1$ & $0.2 \pm 0.1$ & $0.4 \pm 0.1$ \\
\hline & RCA & $0.6 \pm 0.2$ & $0.5 \pm 0.1$ & $0.6 \pm 0.1$ & $0.7 \pm 0.1$ & $0.7 \pm 0.1 *$ & $0.7 \pm 0.1 *$ \\
\hline & LCX & $0.4 \pm 0.1$ & $0.8 \pm 0.2$ & $1.0 \pm 0.3^{*}$ & $0.7 \pm 0.2$ & $0.9 \pm 0.2$ & $1.1 \pm 0.3^{*}$ \\
\hline
\end{tabular}

any lead), one $T_{\text {end }}$ (the latest reliable T-wave end) and one $T_{\text {peak }}$ (the median value of 15 leads) with an outlier protection rule [8]. Then to quantify and analyze SCR, each $i^{t h}$ beat of ECG was restricted to the repolarization windows. These windows were calculated as the earliest and latest reliable T-wave onset and end between 15 leads. Afterwards SVD was applied on the ECG signals windows and SCR series were calculated. The SVD allowed the identification of a set of 15 values that represent the relative magnitude of the spatial components of repolarization. The contribution of second eigenvalue related to first was calculated as:

$$
T_{\mathrm{C}-2 \mathrm{D}, \mathrm{i}}=\frac{\lambda_{2, i}}{\lambda_{1, i}} * 100
$$

the ratio of the second eigenvalue to the total energy as:

$$
T_{\mathrm{C}-2 \mathrm{nd}, \mathrm{i}}=\frac{\lambda_{2, i}}{\sum_{j=1}^{15} \lambda_{j, i}} * 100
$$

and the T-wave residuum to quantify the relative contribution of non-dipolar components as:

$$
T_{\mathrm{WR}, \mathrm{i}}=\frac{\sum_{j=4}^{15} \lambda_{j, i}}{\sum_{j=1}^{15} \lambda_{j, i}} * 100
$$

$T_{\mathrm{C}-2 \mathrm{D}}$ and $T_{\mathrm{C}-2 \mathrm{nd}}$ were calculated in a different way than in [3], where a root mean square factor was used in the original definition.

\section{Results}

We calculated TRD and SCR series during control and ballon inflation in PTCA. For the sake of robustness, we applied a median filtering with a windows size of $7.5 \mathrm{sec}$ on the $T_{\mathrm{W}}, T_{\mathrm{PE}}, T_{\mathrm{C}-2 \mathrm{D}}, T_{\mathrm{C}-2 \mathrm{nd}}$ and $T_{\mathrm{WR}}$ series and characterized 6 different time instants. Control situation $\left(D_{\text {CONTRoL }}\right)$ associated to the median value of 5 minutes just before the start of occlusion, dispersion during occlusion start $\left(D_{\mathrm{PTCA}}^{\mathrm{OS}}\right)$ associated to the median value of the first $7.5 \mathrm{sec}$ of occlusion, dispersion during the first minute $\left(D_{\mathrm{PTCA}}^{\mathrm{O}}\right)$ associated to the median of $7.5 \mathrm{sec}$ centered around the first minute, the second $\left(D_{\mathrm{PTCA}}^{\mathrm{O} 2}\right)$, the third $\left(D_{\mathrm{PTCA}}^{\mathrm{O} 3}\right)$, for their respective minutes, and finally the end of the occlusion $\left(D_{\mathrm{PTCA}}^{\mathrm{OE}}\right)$ associated to the $7.5 \mathrm{sec}$ just before the end. We compared statistically $D_{\text {Control }}$ versus $D_{\text {PTCA }}^{\mathrm{OS}}, D_{\mathrm{PTCA}}^{\mathrm{O} 1}, D_{\mathrm{PTCA}}^{\mathrm{O} 2}, D_{\mathrm{PTCA}}^{\mathrm{O} 3}$ and $D_{\text {PTCA }}^{\mathrm{OE}}$ respectively using two-tailed Wilcoxon and MannWhitney tests. The average time-course of $T_{\mathrm{PE}}$ and $T_{\mathrm{C}-2 \mathrm{D}}$ are plotted in Fig.1, showing the responses of both parameters refered to: (a) and (c) the onset of ischemia and (b) and (d) reperfusion. In Figs. 1(a) and 1(c), all records were temporally aligned taking the balloon inflation as reference, while on Figs. 1(b) and 1(d) the reference for alignment 
is the balloon release. Higher values of $T_{\mathrm{PE}}$ were observed from start to end of PTCA procedure. In contrast, $T_{\mathrm{C}-2 \mathrm{D}}$ increased monotonically, exhibiting the trend to be greater at time coursed of PTCA. The statistical results are presented in Table 1. Results have shown statistically significant differences in $T_{\mathrm{PE}}$ during $D_{\mathrm{PTCA}}^{\mathrm{Os}}, D_{\mathrm{PTCA}}^{\mathrm{O} 1}, D_{\mathrm{PTCA}}^{\mathrm{O} 2}, D_{\mathrm{PTCA}}^{\mathrm{O} 3}$ and $D_{\text {PTCA }}^{\text {OE }}$ when were compared with $D_{\text {CONTROL }}$ in RCA and LAD artery occluded. Also in general, the largest value of $T_{\mathrm{C}-2 \mathrm{D}}$ and $T_{\mathrm{C}-2 \mathrm{n}}$, in mean, were found in RCA, LAD and LCX arteries occluded, however, few significant differences were found for $T_{\mathrm{wR}}$ and $T_{\mathrm{w}}$.

\section{Discussion and conclusions}

We hypothesized that the increase of TRD may denote differential shortening of APD in some myocardium areas, reflecting the times at which the APD finish and, therefore, it could be considered as an index of VRD. The results showed that $T_{\mathrm{w}}$ does not change significantly possibly due to the difficulties of $T_{\text {onset }}$ delineation during ST elevation. $T_{\mathrm{PE}}$ index solved that trouble due to the stability of $T_{\text {peak }}$ in contrast to $T_{\text {onset }}$ delineation. The increase of $T_{\mathrm{PE}}$ observed in our results is compatible with increased transmural dispersion at early ischemia stages (slight reduction in the endocardial APD while the epicardial APD is reduced in a larger proportion).

$T_{\mathrm{C}-2 \mathrm{D}}$ and $T_{\mathrm{C}-2 \mathrm{nd}}$ provide information that can be visualized by analogy as the shortening and lengthening axes of the 2D T-wave loop and provides an estimate of the relative roundness or flatness of the T-wave loop. Then, it could also be assumed that the changes of $T_{\mathrm{C}-2 \mathrm{D}}$ and $T_{\mathrm{C}-2 \mathrm{nd}}$ reflect a rise in global heterogeneity of repolarization and, hence, determine a dynamic increase in the T-wave complexity during PTCA evolution. $T_{\mathrm{wR}}$ does not significantly change suggesting that increase of VRD during ischemia does not modify the validity of the dipolar assumption. This may indicate the unability of $T_{\mathrm{WR}}$ parameter to reflect regional heterogeneity of repolarization.

It is important to evaluate this results relative to $T_{\mathrm{C}-2 \mathrm{D}}$ and $T_{\mathrm{C}-2 \mathrm{nd}}$ in view of the fact that ischemia largely affects the ST segment, and even the eigenvalues are computed from onset of T wave (after ST segment finish) this onset is largely affected by ST elevations. Then the evolution of this Twave complexity indexes can be affected by the fact that the window where they are computed changes over time, and also the influence of ST changes that go well into the T-wave [5]. So it is not clear whether this changes are because of VRD increase of because ischemia modifications of ST-T complex morphology, or even because a mixture of both.

The $T_{\mathrm{PE}}$ is more clearly physiological related to VRD increase, and according to the results the change is reached at the first instants of ischemia and remains until the ischemia disappears (Fig.1).
The results presented in this work encourage the undertaking of deeper studies to correlate and better understand the relationship between APD myocardial dispersion with acute ischemia.

\section{Acknowledgements}

This work was supported by projects TEC2007-68076C02-02 from MCYT/FEDER, Spain, and by a personal grant to PD Arini from IAM-CONICET (PIP 5272), Argentina.

\section{References}

[1] Lukas A, Antzelevitch C. Differences in the electrophysiological response of canine ventricular epicardium and endocardium to ischemia: Role of the transient outward current. Circulation 1993;88:2903-2915.

[2] Arini PD, Bertrán GC, Valverde ER, Laguna P. T-wave width as an index for quantification of ventricular repolarization dispersion: Evaluation in an isolated rabbit heart model. Biomedical Signal Processing and Control 2008;3:67-77.

[3] Priori S, Mortara D, Napolitano C, Diehl L, Paganini V, Cant F, Cant G, Schwartz P. Evaluation of the spatial aspects of $\mathrm{T}$ wave complexity in the long-QT syndrome. Circulation 1997;96:3006-3012.

[4] Malik M, Acar B, Gang Y, Yap YG, Hnatkova K, Camm AJ. QT dispersion does not represent electrocardiographic interlead hetereogeneity of ventricular repolarization. J Cardiovasc Electrophysiol 2000;11:835-843.

[5] García J, Lander P, Sörnmo L, Olmos S, Wagner G. Laguna P. Comparative study of local and Karhunen-Loève-Based ST-T indexes in recording from human subjects with induced myocardial ischemia. Computer and Biomedical Research 1998;31:271-292.

[6] Moody GB, Mark RG. Development and evaluation of a 2 lead ECG analysis program. Computers in Cardiology 1982; $39-44$.

[7] Martínez JP, Almeida R, Olmos S, Rocha AP, Laguna P. A wavelet-based ECG delineatior: Evaluation on standard databases. IEEE Transactions on Biomedical Engineering 2004;51(4):570-581.

[8] Laguna P, Jané R, Caminal P. Automatic detection of wave boundaries in multilead ECG signals: Validation with the CSE. Comp Biomed Res 1994;27:45-60.

Address for correspondence:

Pedro David Arini

Instituto Argentino de Matemática, CONICET

Saavedra 15, $3^{e r}$ piso, 1430, Ciudad Autónoma de Buenos Aires, Argentina

pedroarini@yahoo.com.ar 